\title{
Téoros
}

Revue de recherche en tourisme

\section{Perspectives d'avenir des croisières longue durée sur le Saint-Laurent}

\section{Luc Tittley}

Volume 20, numéro 1, printemps 2001

Mers et littoraux

URI : https://id.erudit.org/iderudit/1071903ar

DOI : https://doi.org/10.7202/1071903ar

Aller au sommaire du numéro

Éditeur(s)

Université du Québec à Montréal

ISSN

0712-8657 (imprimé)

1923-2705 (numérique)

Découvrir la revue

Citer cet article

Tittley, L. (2001). Perspectives d'avenir des croisières longue durée sur le

Saint-Laurent. Téoros, 20(1), 4-8. https://doi.org/10.7202/1071903ar d'utilisation que vous pouvez consulter en ligne.

https://apropos.erudit.org/fr/usagers/politique-dutilisation/ 


\section{Perspectives d'avenir des croisières longue durée sur le Saint-Laurent}

\section{Luc Tithley}

Avant l'avènement de l'avion, les liaisons entre les continents, aw-dela des mers et des océans, se faisaient par bateau : des navires marchands et des paquebots. On embarquait dans un port et, après un intermede en mer souvent long, on debarquait dans le Nouveau Monde, sur le Vieux Continent ou dans quelque colonie lointaine. Les côtes étaient des lignes d'horizon marquant le point de départ et le point d'arrivee. Alors, la destination primait sur le trajet. Celle epoque est aujourd'hui révolue.
Q ertes, le transport de passagers par bateau existe toujours sur les mers parsemées d'îles et les côtes difficiles d'accès de certains littoraux océaniques. Mais sur ces côtes souvent riches en paysages. le trajet l'emporte sur la destination dans la motivation de la clientèle aujourd'hui dominée par les touristes. Qu'il s'agisse de navires de ravitaillement desservant les côtes de l'Alaska ou de la Norvège ou de traversiers reliant les îles greeques, les bâtiments adoptent des trajets conçus pour le plaisir des passagerset ils prennent des airs de paquebots. Ce sont maintenant des navires de croisières, grands et petits, à voile ou à moteur, qu'on voit se prélasser dans les mers chaudes ou s'aventurer dans les eaux les plus reculées. Un peu partout, le but du voyage en mer n'est plus seulement de relier le port de destination, mais bien plutot le trajet pour s'y rendre qui permet d'apprécier un paysage, d'explorer ses richesses, de découvrir un pays.

Le fleuve Saint-Laurent, son estuaire, son golfe, l'ensemble du littoral atlantique du Canada font partie de ces régions capables d'attirer les touristes en mal de paysages inusités. Depuis une dizaine d'années, l'offre de croisières-excursions s'y est multipliée, élargissant les possibilités d'apprécier l"environnement unique d'un des grands fleuves du monde.

La grande popularitế de ces excursions de quelques heures permet-elle d'envisager, en ce début de millénaire, un retour des croisières longue durée du début du siècle dernier, illustrées de façon si romantique par les bateaux blanes de la Canadian Steamship Lines (CSL) ? Les enjeux sousjacents au développement de croisières sut le littoral est du Canada sont explorés sommairement dans les lignes qui suivent. La réflexion qui est proposée aborde les perspectives d'avenir de ce produit tou= ristique, autrefois très populaire, à la lumière des tendances actuelles qui marquent l'offre et la demande mondiales. Elle est centrée sur les croisières avec coucher at bord".

\section{La disparition des bateaux blancs}

Ceux qui ont plus de cinquante ans ont au moins un vague souvenir des bateaux blancs. Le transport de passagers sur le fleuve est apparu avec les premiers établissements humains, mais, à partir de 1830 environ, plusieurs compagnies de navigation mettent en service des navires concus spécifiquement pour la croisière ${ }^{2}$. Ces navires connaîtront un frane succès pendant plus d' un siècle. Ils joueront un rôle, qu'on qualifierait aujourd'hui de structurant, dans le développement de l'infrastructure touristique du Québec en bordure du Saint-Laurent. La dépression des années trente provoquera le déclin des croisiêres, puis la disparition des bateaux blanes.

L'automobile, devenant de plus en plus accessible et performante pour \& voir du pays *, remplace le navire à vapeur dans la découverte du paysage touristique québécois. Dès les années cinquante, le tourisme de circuit se cantonne pour ainsi dire sur la terre ferme. Les. Québécois se contentent de voir le fleuve de la côte, plutôt que de voir la côte à partir du fleuve.

Les annees quatre-vingts voient le contact avec le Saint-Laurent se rétablir progres- 


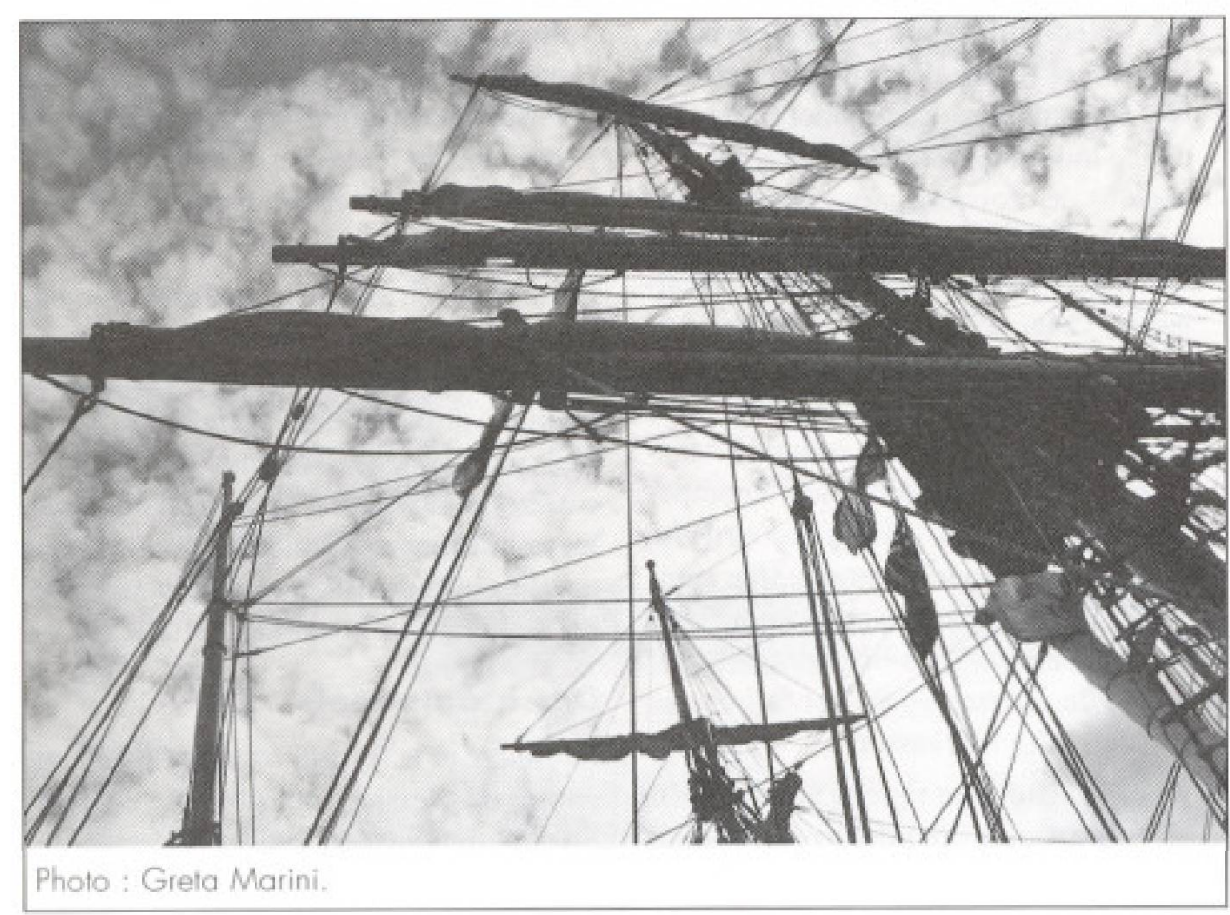

sivement, grâce à des $\alpha$ fenêtres sur le fleuve * ouvertes dans de nombreuses villes riveraines, grandes et petites. Ces interventions volontaristes permettent de renouer avec la richesse de l'environnement fluvial, d'en inverser la dégradation et d'apprécier la diversité de ses paysages. Le Québécois en vacances se résout même à quitter les berges pour s"aventurer au large. Le fleuve reprend sa veritable place dans le patrimoine collectif et amorce son repositionnement parmi les grands attraits touristiques du Québec.

\section{Le Saint-Laurent en vedette}

Le fleuve Saint-Laurent baigne, sur plus 1600 kilomètres, les côtes intérieures du Québec ; son estuaire est vaste et s'ouvre sur un golfe plus vaste encore. Le littoral atlantique, comme certains tronçons du fleuve, est dentelé de baies, d'anses, de fjords, de caps, de presqu"îles et de péninsules et il est peuplé d'innombrables îles. L'ensemble forme un espace varié et unique de contact entre la mer et le continent.

Au cours des cinq dernières années, pas moins de sept ouvrages grand public (Croteau, 1995 ; Frances-Schurr, 1996 ; Ouellet et Vézina, 1997 ; Potvin, 1984 ; Ouellet, 1998 ; Franck, 2000 ; Matte et Pellerin, 1999) ont été consacrés aux paysages du Saint-Laurent, à ses îles, à sa faune aquatique, à ses phares, à ses modes de vie et ă son histoire. Et que dire des sections de mieux en mieux documentées dans les guides de voyage publiés ici (Ulysse) ou a l ${ }^{+}$etranger (Michelin, Feudor, Gallimard et autres), ou encore des albums de photos de Mia et Klaus ou des monographies régionales de l'Éditeur officiel du Québec sur la Gaspésie ou la CôteNord ? Les reportages et les émissions thématiques se multiplient à la radio comme à la télévision. Des revues comme GEO, Continuité, Fonces, Géo Plein-Air et bien d'autres s'y intéressent aussi.

Le Saint-Laurent n'est plus un sujet de conversation réservé aux nostalgiques, aux capitaines, aux géographes, aux biologistes ou aux poètes. Il fait partic aujourd'hui du quotidien des Québécois : de leurs projets de vacances comme de leur liste de livres à offrir en cadeau. Qui plus est, il a rejoint les * grands espaces sauvages $*$ et la $*$ cabane au Canada $\gg$ dans I'imaginaire touristique de nos cousins français et des autres voyageurs de passage.

L'engouement pour l'observation des baleines $n$ 'est pas étranger à ce regain d'intérêt. En effet, le spectacle de ces mammiferes marins a largement contribue à faire redécouvrir aux Québécois et aux touristes étrangers le plaisir d'une excursion sur l'eau. Une véritable industrie est née, offrant un nouveau point de vue sur le pays et la richesse du fleuve qui en forme l'épine dorsale : les mammiferes marins. certes, mais aussi les rapides de Lachine, le port de Montréal, le cap Diamant, le fjord du Saguenay, les fous de Bassan, les phares, les îles, etc. Les membres de cette industrie, regroupés dans l'Association des croisières-excursions du Quebec, ont transporté 909085 passagers au cours de la saison $1996^{7}$ sur des navires et des embarcations de toutes tailles (du Zodiac au traversier modific).

\section{Les croisières longue durée}

Chaque annee, environ 35000 passagers embarquent ou debarquent de paquebots dans les ports de Montréal et de Québec. Plus de la moitié de cette clientèle, attirée par les couleurs automnales, navigue sur le Saint-Laurent en septembre et en octobre sur des navires battant pavillon de grands noms de la croisière océanique comme Commodore, Holland America, Princess, Hapag-Lloyd, Regal, Seabourn. Le Rembrandt de la compagnie Premier Cruises (1 100 passagers) fréquente même le Saint-Laurent, depuis peu, en plein été.

On observe aussi un accroissement du nombre de croisières sur des navires de petite taille conçus pour la navigation côtière et les eaux fluviales (moins de 120 passagers). Des pionniers comme la St. Lawrence Cruise Line ${ }^{4}$ et la compagnie Desgagnés s ont été rejoints par American Canadian Caribbean Cruise Line ${ }^{6}$ et Clipper Cruise Line7. Depuis l'été 2000, une entreprise française (la Cie des îles du Ponant) a mis en service un nouveau navire de croisière fluviale, le Levant (95 passagers), entre Montréal et Saint-Pierre-etMiquelon.

La position de la destination CanadaNouvelle-Angleterre demeure cependant modeste a l'échelle mondiale (moins de $1 \%$ de l'offre et sept fois moins que l'Alaska), malgré la proximité du plus grand pays émetteur de croisiéristes (les Nord-Américains représentent environ $80 \%$ de la demande mondiale). Préoccupés par cette situation, les intervenants 
touristiques ont formé récemment l'Association des croisieres du Saint-Laurent en vue de coordonner les efforts de promotion requis pour améliorer cette situation.

\section{Des indicateurs favorables}

La naissance fort attendue de 1'Association survient alors que les tendances mondiales sont de plus en plus favorables. au developpement des croisières longue durée :

- Le marché des croisières est toujours important et en forte croissance. Environ, 7 millions de personnes, dont 5,83 millions de Nord-Américains, choisissent le produit croisière pour leurs vacances $^{8}$. Le taux de croissance annuelle moyen est de l'ordre de $9 \%$ depuis les 20 dernières années. Il s'est maintenu au-dessus de $8,5 \%$ en 1999. Cette situation, combinée à une évolution sociodémographique favorable (encore les baby boomers) et à un taux élevé de répétition, assurera une croissance soutenue de la demande pour plusieurs annees. encore.

- Cette forte demande pousse les entreprises de croisiêres à augmenter la capacite d'accueil (plus de navires, des navires plus gros) sur les destinations bien établies (destinations soleil, sites spectaculaires, lieux d'histoire). Elle incite aussi à clargir la gamme des produits (destinations nouvelles, croisières thématiques, croisieres courtes), afin de satisfaire la clientèle d'habitués et d"attirer de nouveaux segments de marché (clientele jeune et scolarisec).

- Malgré la position dominante des NordAméricains sur le marché, on constate une algmentation de $100 \%$ de la demande provenant de l'Europe et de l'Asie, des marchés en émergence à très grand potentiel pour les années à venir. Le developpement de l'offre de destinations européennes (mer du Nord, côtes scandinaves, Baltique, Méditerranée) y est sans doute pour quelque chose. Il en est de même pour les circuits exotiques. notamment dans le bassin du Pacifique, ainsi que 1'Arctique et 1'Antarctique.

- Les destinations soleil dominent toujours le marché des croisières, mais les efforts de diversification de l"offre rejoignent les valeurs du jour : l"écotourisme, $\mathrm{I}^{+}$aventure légère, l'exotisme confortable, la découverte du monde. Cette tendance lourde influence l'émergence de régions de croisieres nouvelles : l'Antarctique et les îles Falkland et Shetland, les fjords de Norvège et l'archipel Svalbard, la mer Baltique, le Groenland et l'Arctique, la presqu'île de Californie sur la côte occidentale du Mexique et les grands fleuves du monde conme l'Anazone. De plus, au moins quatre compagnies spécialisées offrent aujourd'hui des circuits autour du monde (par troncons), dont la compagnie allemande Phonix Reiser GmbH, propriétaire actuel du Maxim Gorki qui fait escale à Montréal à l'occasion.

- La diversification touche aussi les navires. Plusieurs opérateurs optent pour des navires petits, à l'opposé des mégapaquebots, afin d'offrir un espace convivial mieux adapté à l'exploration de plans d'eau et des rivages difficiles d'accès: pocket ships de luxe (ex. Seabourn), navires à coque brise-glace (ex. Bergen Line), à faible tirant d'eau (ex. Clipper), grands et moins grands voiliers (ex. Windstar), etc. D'autres choisissent de rehabiliter des paquebots anciens, romantiques certes, mais aussi moins chers (ex. World Explorer).

- Les * concepts $*$ de vacances ont envahi la croisiere. Au-dela de la destination, on commercialise aujourd'hui un theme (nature, histoire, musique, gastronomie,

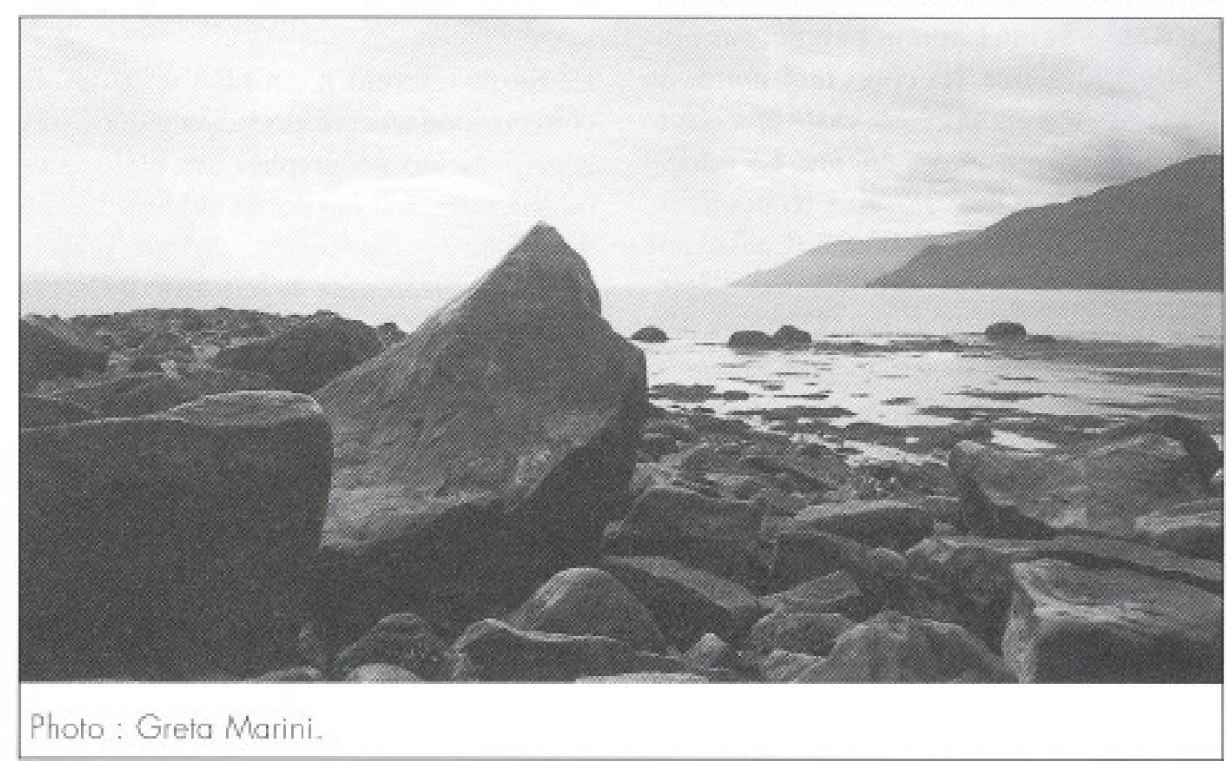

etc.) ou encore une ambiance (le clinquant des méga-paquebots, l'atmosphère rétro de certains navires anciens, le raffinement feutré de grands yachts haut de gamme ou encore l'aventure spartiate sur des navires d'expedition).

En somme, on assiste à une forte segmentation du marché de la croisière. Cette tendance de fond est à l'avantage de destinations qui offrent des attraits distinctifs réels, notamment en matière de croisières cotiêres et fluviales. Dans un tel contexte, les conditions apparaissent réunies pour améliorer la position, encore marginale, du fleuve et du littoral atlantique.

\section{Des atouts réels}

Le fleuve et le littoral forment une destination de croisière qui possède de nombreux atouts susceptibles d'intéresser les amateurs de croisières. Ils peuvent être regroupés en deux familles:

- La première concerne les entreprises de croisiètes internationales : celles dont les paquebots géants accostent à Montréal et à Québec. Pour elles, les deux grandes villes de l'itinéraire sont attirantes parce qu'elles offrent le confort nord-américain, agrémenté d'un soupcon d'exotisme europécn. Américains et Européens y trouvent leur compte. Montréal et Québec offrent aux croisiéristes des valeurs sûres : dépaysement culturel léger, animation originale de calibre international, attraits touristiques, sites d'intérêt, etc. Elles assurent aussi 
aux exploitants les services attendus d'un port d'embarquement ou d'un port d'escale : desserte aérienne, infrastructure d'accueil, approvisionnement, etc.

Même si les croisières se font surtout en septembre et en octobre pour profiter des couleurs automnales ${ }^{9}$, on constate un nouvel intérêt pour l'exploitation de navires durant toute la saison estivale. Les operateurs qui s'y risquent tablent à la

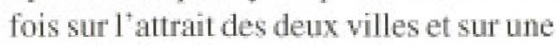
appréciation plus pointue des paysages variés le long du trajet ${ }^{10}$, notamment celui de la pointe gaspésienne, du fjord du Saguenay et des côtes en amont.

- La deuxième famille d'atouts concerne les croisières côtières et fluviales, très près des tendances contemporaines liées au tourisme d'aventure et à la découverte de la nature. Les possibilités offertes dans ce domaine sont énormes et peu exploitées commercialement ${ }^{11}$. Plusieurs des paysages côtiers du fleuve et du golfe du Saint-Laurent, tout comme les côtes de Terre-Neuve et du Labrador, sont difficiles d'accès, mais non moins spectaculaires.

Le Saint-Laurent et son embouchure soutiennent facilement la comparaison avec plusieurs destinations de plus en plus populaires comme les côtes de l'Écosse, de la Norvège ${ }^{12}$ et de la Finlande, la côte occidentale canado-américaine $^{13}$, ou encore la côte du sud de 1'Argentine et le continent antarctique voisin ${ }^{14}$.

Le changement des saisons qui renouvelle les paysages est aussi un atout qu'on apprend tout juste à valoriser. L'hiver québécois, longtemps considéré comme un plaisir pour consommation locale, est en voie de devenit un produit d'intérêt universel. Les croisières d'hiver sont toutefois impensables sur le Saint-Laurent ${ }^{15}$. En effet, le froid, les glaces et les conditions de navigation, ajoutés à l'exiguitté du marché, à la rareté des navires adaptés et aux coûts d'exploitation très élevés, sont des obstacles encore incontournables à la viabilité des croisières d'hiver. Même les navires qui approvisionnent les villages côtiers regagnent les ports du Sud pour l'hiver.

\section{Les défis}

Malgré la richesse des ressources, le développement d'une industrie de la croisière fluviale sur le Saint-Laurent, ou encore sur les côtes de Terre-Neuve et du Labrador, n'est pas évident. Les défis sont nombreux et importants :

- Les conditions climatiques qui prévalent au Québec limitent la saison à un maximum de 120 jours par année. La rentabilité de l'exploitation est impossible sur une si courte période, ce qui impose de déplacer les navires vers des eaux clémentes lé reste de l'année. Les entreprises doivent donc avoir les compétences et le savoir-faire pour evoluer sur plus d'un théâtre d'opération. C'est le cas de toutes les entreprises qui exploitent des navires de croisières, grands et petits, dans des régions comme les côtes de l' Alaska, la mer Baltique, la côte de la Norvège ${ }^{16}$ ou encore l $^{+}$Antarctique.

- Aujourd'hui, le Saint-Laurent est fréquenté surtout par des paquebots étrangers de grande taille qui sont le véhicule privilégié des croisiềres de masse. Ces navires exigent des installations et des services portuaires relativement sophistiqués. Les croisières spécialisées font appel à des navires petits, à faible tirant d'eau, donc capables de naviguer sur des plans d'eau peu profonds et se contentant de structures maritimes minimales. $\mathrm{Ce}$ pendant, le nombre de mouillages protégés et de quais publics, déjà peu nombreux sur le Saint-Laurent, est en diminution constante à cause du délestage de structures maritimes entrepris par Pêches et Océans Canada. Cette situation réduit les points d'accès aux attraits terrestres ainsi que les abris pour les plus petits navires. Une réflexion sérieuse s'impose afin de préserver un réseau minimal si l'on veut développer un jour le potentiel de croisières côtières et fluviales.

- Le niveau de confort de ces petits hôtels flottants capables de naviguer dans des régions très isolées peut être spartiate (ex. Canadian Empress, Nordic Express), moyen (ex. Echo des Mers) ou encore très luxueux (ex. Seabourn Pride ou Hanseatic). Le prix de la croisière est à l'avenant. Dans tous les cas, le coutt du navire et les frais d'exploitation sont des intrants importants dans le calcul de la rentabilité. Au Canada, la législation sur la construction et l'importation temporaire des navires, le cabotage et les equipages pose un défi énorme aux promoteurs. On compte seulement deux entreprises canadiennes à l'ruuvre au Québec: St.-Lawrence Cruise Line (Canadian Empress), basée à Kingston, et Écomertour de Rimouski (Écho des Mers). Le cadre réglementaire est plus favorable aux opérations basées sur l'importation temporaire de navires par des entreprises étrangères. La plupart sont américaines ${ }^{17}$, à l'image de l'ensemble de l'industrie. La voie la plus facile du développement passe par des partenariats adaptés aux conditions locales avec des entreprises étrangères bien implantées.

- Le port d'embarquement-débarquement est une composante critique de l'équation de la rentabilité des croisières. Celui-ci doit offrir des liaisons aériennes régulières avec les marchés émetteurs. Il doit aussi être situé à distance raisonnable des principaux attraits du ou des circuit(s) offert(s). Aucun port ne peut répondre aux besoins de toutes les entreprises. Par exemple, le port de Québec est bien situé pour des itinéraires vers l'estuaire, celui de Gaspé pour le golfe et celui de Saint-Jean, Terre-Neuve, pour les circuits vers la côte du Labrador. Le port de Montréal est mieux situé pour s'inscrire dans des circuits axés sur les Mille-Îles, l'Outaouais et les canaux historiques américains et ontariens. Le défi est d'éviter la concurrence stérile entre ports d'une même région de croisière. En conséquence, la création de I'Association des croisières du SaintLaurent est un pas dans la bonne direction.

- La commercialisation des produits de croisières longue durée sur le SaintLaurent exige de la part des entreprises une stratégie et une équipe de vente adaptées à la capacité d'accueil et aux marchés ciblés. Le défi est de première importance pour les entreprises à faible capitalisation. Internet fournit certes des 
possibilités nouvelles et très efficaces pour commercialiser directement le produit $^{13}$. Or, la création et la gestion de forfaits dépassent souvent la capacité organisationnelle de l'entreprise. L'intégration dans des réseaux établis, par alliance avec des grossistes ou des voyagistes crédibles, constitue souvent un choix plus rentable.

\section{Conclusion}

Le Québec connaît des succès remarqués en matière de tourisme urbain ; les entreprises culturelles locales s'affirment sur les scènes internationales alors que l'axe de promotion culture-nature est porteur dans un nombre croissant de marchés étrangers, le tourisme hivernal est en pleine affirmation. L'industrie des eroisièresexcursions atteint la maturite. Le moment est maintenant venu d'attirer l'attention sur les croisières longue durée sur le SaintLaurent, un des grands potentiels encore à développer. La multiplication des produits doit faire partie des objectifs à poursuivre. Les défis sont importants, mais les ressources sont riches et le marché porteur.

\section{Luc Tittley, geographe et urbaniste,} est consultant en gestion de projets de développenent et d'aménagement touristique. Il a dirigé plusieurs études sur les croisières sur le Saint-Laurent ${ }^{19}$.

\section{Notes}

1 Certains des propos qui suivent peuvent s'appliquer aux croisières avec coucher à terre.

2 Pour un aperçu de l'histoire des croisières sur le Saint-Laurent, on peut se référer à l'article de Tittley (1995).

3 Source : Profil de l'industrie des croisièresexcursions au Québec en 1996, réalisé par l'auteur pour le compte de l'Association des croisières-excursions du Québec. Les données statistiques sur cette industrie n'ont pas été mises à jour depuis 1996. Toutefois, certains facteurs comme les conditions météorologiques estivales, l'évolution de la fréquentation des régions touristiques clés, ainsi que la réduction du nombre d'entreprises de croisières portent à croire que le nombre de passagers transportés aurait êté d'environ un million en 2000.
4 Le Canadian Empress est exploité à partir de Kingston, Ontario.

5 Cette compagnie exploite le Nordic Express, cargo mixte qui assure la desserte hebdomadaire des communautés de la Côte-Nord d'avril au début de janvier; depuis Rimouski.

6 Depuis plusieurs années cette entreprise, qui est basée à Warren, Rhode Island, affecte le Grande Caribe et le Grande Mariner au Saint-Laurent et à la côte Atlantiqué pendant l'été.

7 La Clipper Cruise Line de St-Louis, Missouri, fait partie du groupe Kuoni Travel. Elle exploite des navires de petite taille sur presque toutes les mers du monde. Le Nantucket Clipper (100 passagers) est affecte au fleuve et au golfe du SaintLaurent pendant l'êté.

8 Selon la Cruise Line International Association (CLIA).

9 A la faveur du repositionnement saisonnier des eaux europennes vers les mers chaudes.

10 Les circuits de croisières internationales incluent habituellement la côte de l'Atlantique jusqu'à Boston ou New York.

11 Sauf par l'Écho des Mers et le Nordic Express.

12 Bergen Line offre des croisières à l'année dans les fjords et dans l' archipel Svalbard at bord de onze navires mixtes pouvant transporter de 200 à 490 passagers.

\section{Bibliographie}

Croteau, André (1995), Les ûles du Saint-Laurent, Trécarré, Montréal, $189 \mathrm{p}$.

Daniel Arbour \& Associés (1993), Pland'action du Comité des croisières du Pont de Montréal.

Francès, Patrick, et Schurr, Jean-Eudes (1996), Le Québec au fil du Saint-Laurent, Éditions du Chêne, Paris, 189 p.

Franck, Alain (2000), Naviguer sur le flewve au temps passe, Les Publications du Québec, Quebec, $104 \mathrm{p}$.

Matte, Gilles, et Gilles Pellerin (1999), Carnets du Saint-Laurent, Editions 400 coups, Montréal.

Ouellet, Danièle, et René Vézina (1997), La passion da Saint-Laurent a a la decouverte du grand flewve, Multimondes, Montréal, 217 p. Ouellet, Marie-Claude (1998), Le fleuve SaintLaurent, Éditions de l'Homme, Montréal.

Potvin, Damase (1984), Le Saint-Laurent et ses iles. Histoire, légendes, anecdotes, description, topographie, Montreal, Editions Lémeac.
13 La côte ouest du Canada et de l'Alaska constitue la deuxième destination de croisière la plus fréquentée au monde. Toutes les classes d'entreprises de croisières y naviguent pendant l'été.

14 Pendant l'été austral, pas moins de quatre entreprises internationales offrent des croisières dans les eaux de l'Antarctique à partir du port de Ushuaia en Argentine : Lindblad Expeditions (autrefois Special Expeditions), Clipper Cruise Line (Clipper Adventurer), Radisson Seven Seas (Hanseatic) et Abercrombic \& Kent (Explorer).

15 Le Nordic Express suspend ses opérations de janvier à avril.

16 La compagnie norvégienne Bergen Cruise Line constitue une exception à la règle. Elle exploite, à longueur d'année, onze navires brise-glace qui approvisionnement tous les villages côtiers. Chacun de ces navires est conçu pour accueillir de 100 à 200 passagers, dont une majorité de touristes.

17 Clipper Cruise Line et American Canadian Caribbean Cruise Line pour les navires de petite taille; Holland America, Premier et autres pour les navires de grande taille.

18 On peut passer par lé portail www.travellibrarycom/cruises pour avoir accès à la plupart des entreprises de croisic̀re.

19 The Economic Planning Group, et Arbour \& Associes, 1991 ; Arbour \& Associes, 1993 ; Tituley, 1997.

The Economic Planning Group, et Daniel Arbour \& Associes (coauteurs) (1991), Anabse de l'état de l'industrie des croisières et des excursions sur les Grands Lacs, le fleuve SainLaurent et leurs voies navigables de jonction, mars.

Tittley, Luc (1997), Services conseil en tourisme et amenagement, Profil de l'industrie des croisieres-excursions au Québec en 1996. Association des croisières-excursions du Québec.

Tittley, Luc (1995), \& Les croisières sur le SaintLaurent... un peu d'histoire *, Téoros, 14 (2). 